\title{
Identification of Source Emission Rate in a Multi-zone Building Using Regularization Approach
}

\author{
Xiaoran Liu ${ }^{1}$, Fei $\mathrm{Li}^{1 *}$, Jinxiang Liu ${ }^{1}$, Hao Cai ${ }^{1}$, Junyi Zhuang ${ }^{1}$ \\ ${ }^{1}$ Department of HVAC, College of Urban Construction, Nanjing Tech University, Nanjing, China
}

\begin{abstract}
It is critical to identify the contaminant source location and emission rate as soon as possible and take proper actions to ensure people safe in the buildings. In this study, we built Markov Chain model and used Tikhonov regularization to inverse the source emission rate in a multi-zone building. We further investigated the effects of the sensor location selection, time resolution $(5 \mathrm{~s}, 10 \mathrm{~s}, 20$ s) and methods for choosing the regularization parameter (L-Curve, GCV, Quasiopt) on the results of inverse calculation. The results show that for Sensor 1, the average percentage of the points below the threshold (relative error is 10\%) is $98.75 \%$, and that of Sensor 2 is $78.66 \%$, which indicates the sensor more closed to the source can provide more accuracy inverse emission rate. The results also show that different parameter selection methods have different favourite time resolutions. When the time step is $20 \mathrm{~s}$, the relative errors of the L-Curve method are less than $8 \%$, and the relative errors of the Quasiopt method are below $2 \%$ under $5 \mathrm{~s}$. The GCV method performed best when time step is $5 \mathrm{~s}$, and the number of nodes with a relative error less than $10 \%$ in the GCV method accounted for $94.68 \%$.
\end{abstract}

\section{Introduction}

People spend almost $90 \%$ of their time in indoors (Chen and Zhao, 2011). Therefore, the enclosed environment safety is significantly important for peoples' health. Primary indoor pollutants that threaten human health can be classified into three categories according to their threat severity. The light threat sources include occupants, building materials, and fuel combustion, which can cause allergy, asthma symptoms, and Sick Building Syndrome (SBS). The medium threat sources include virus, bacteria, and fungus, which can cause infectious diseases (e.g. SARS (Li et al., 2010)). The serious threat sources include toxic chemicals and bio-aerosols. In extreme conditions, they can be used as biochemical weapons for terrorist attacks such as the Tokyo subway attack in 1995 (Alexander and Klein, 2003). In order to build safe and healthy built environment, it is critical to identify the source location and release profile fast and accurately in the case of an airborne contaminant release.

The source term estimation (STE) methods can be classified into two major types: passive fixed-sensor method and active olfactory method. For the active olfactory method, the contaminant sensors are usually incorporated with a mobile platform with multi-robots
(Chen et al., 2017; Feng et al., 2017; Zou et al., 2009). The most common STE method is the passive fixedsensor method including forward, backward, and adjoint probabilistic solution

A forward method stores all potential release source terms (location and strength) that are pre-simulated as a database, and then search for a solution that marches the measured data from sensors from an efficient search algorithm such as Bayesian Monte Carlo algorithm (Sohn et al., 2002) or intelligent optimization. Another strategy of forward method is to minimize the cost function, which presents the discrepancy between prediction and measurement (Vukovic et al., 2010; Zheng and Chen, 2011). The simplest algorithm is the classical gradientbased least squares. In order to make their solutions stable, a more robust method is to add a regularization term in the cost function and converts the ill-posed problem to well-posed problem with stable and unique solution (Kathirgamanathan et al., 2004; Tikhonov and Arsenin, 1977; Zhang et al., 2015).

The backward method for source identification is to inverse the transport equation directly, the reverse simulation often involves measuring concentrations from a few locations as boundary or initial conditions. Typical methods include the quasi-reversibility (QR) and the Lagrangian-reversibility (LR) method. The adjoint probabilistic method calculated the backward probability of the source location and release time through solving the adjoint equations. The detailed review could be found in the study of (Hutchinson et al., 2017).

Most studies mentioned above focused on the source identification in a single room. However, the application of the STE method for the multi-compartment public building, such as schools, hospitals, and offices is more meaningful. In this work, we built Markov Chain model and used Tikhonov regularization to inverse the source emission rate in a multi-zone apartment; the performance of three regularization parameter choosing methods under different time resolutions were investigated.

\section{Methodology}

\section{Markov chain model for contaminant transportation in the multi-compartment building}

The Markov chain technique was used to calculate the transient particle transport in multi-zone building. Assuming that the building has $n$ zones, and these zones were referred to as state related to time and location, 
particle was released in certain state, the state either experiences a transition or remains in the initial state in the next time step (Fontanini et al., 2015). Then an $n \times n$ transition probability matrix $\boldsymbol{P}$ with an associated time step can be formed by the probabilities of the state's changing of a particle:

$$
\boldsymbol{P}=\left(p_{i, j}\right)_{(n \times n)}=\left(\begin{array}{cccc}
p_{1,1} & p_{1,2} & \cdots & p_{1, n} \\
p_{2,1} & p_{2,2} & \cdots & p_{2, n} \\
\vdots & \vdots & \cdots & \vdots \\
p_{n, 1} & p_{n, 2} & \cdots & p_{n, n}
\end{array}\right)
$$

where represents the probability of particle's moving from state $i$ to state $j$ in a certain time step, $\Delta t$, in this paper, $\Delta t$ is $5 \mathrm{~s}, 10 \mathrm{~s}$ and $20 \mathrm{~s}$, respectively. The calculation of this matrix is related to the flow field. The transition probability of a particle staying in initial state $i, p_{i, i}$, and the probability of a particle's moving from state $i$ to state $j$ in a time step , $p_{i, j}$, can be calculated by the following equations (Chen et al., 2015):

$$
\begin{gathered}
p_{i, i}=\exp \left(-\sum_{n b} \frac{Q_{i, n b}}{V_{i}} \Delta t\right) \\
p_{i, j}=\frac{Q_{i, j}}{\sum_{n b} Q_{i, n b}}\left(1-p_{i, i}\right)
\end{gathered}
$$

where $Q_{i, n b}$ is the airflow rate from zone $i$ to a neighbouring zone, which can be obtained directly from the CONTAM simulation results. For a steady flow field, the matrix $\boldsymbol{P}$ with an associated time step is fixed.

A particle number vector at the present time (time $k$ ) was defined as follows:

$$
\boldsymbol{N}_{\boldsymbol{k}}=\left(N_{k, 1} N_{k, 2} \cdots N_{k, n}\right)
$$

where $N_{k, i}$ represents the particle number in zone $i$ at time $k$. For the steady flow field with the pulse release source, particle number vector at time $l \Delta t$ can be calculated by Equation (5), and for steady flow field with continuous release source, particle number vector at time $l \Delta t$ can be calculated by Equation (6).

$$
\begin{aligned}
\boldsymbol{N}_{l \Delta t}= & \boldsymbol{N}^{s} \cdot \boldsymbol{P}_{\Delta t}^{l} \\
\boldsymbol{N}_{l \Delta t}= & \sum_{i=0}^{l} \boldsymbol{N}_{i \Delta t}^{s} \cdot \boldsymbol{P}_{\Delta t}^{l-i} \\
= & \boldsymbol{N}_{0}^{s} \cdot \boldsymbol{P}_{\Delta t}^{l}+\boldsymbol{N}_{\Delta t}^{s} \cdot \boldsymbol{P}_{\Delta t}^{l-1} \\
& +\cdots+\boldsymbol{N}_{(l-1) \Delta t}^{s} \cdot \boldsymbol{P}_{\Delta t}+\boldsymbol{N}_{l \Delta t}^{s}
\end{aligned}
$$

where $\boldsymbol{N}^{s}$ is the source particle number vector, $\boldsymbol{N}_{i \Delta t}^{S}$ is the source particle number vector at time $i \Delta t$ in steady flow field with continuous release source, $\boldsymbol{P}_{\Delta t}$ is the transition probability matrix in $\Delta t$ time period, $l$ and $l-i$ are iteration index.

\section{Regularization method for the source strength determination}

The monitored contaminant concentration $\boldsymbol{C}$ and the release rate $\boldsymbol{q}$ can form a linear relationship through the response matrix:

$$
\boldsymbol{C}=\boldsymbol{A q}
$$

where $\boldsymbol{C}$ is the monitored concentration series at different time step, $t_{k}$ is the $k^{\text {th }}$ time step, $\boldsymbol{q}$ is the release rate vector of the source, and $\boldsymbol{A}$ is the response matrix, which describes the cause effect relation between the monitored concentration and the release rate.

The monitored concentration $\boldsymbol{C}$ is provided, while the response matrix $\boldsymbol{A}$ is ill-posed, so the Tikhonov regularization method was introduced to improve the stability of the inverse operation. The Tikhonov regularization method transforms the Equation (7) into a linear least-squares optimization function problem, and the numerical solution can be obtained through a series of transformations:

$$
\boldsymbol{q}=\left(\boldsymbol{A}^{\boldsymbol{T}} \boldsymbol{A}+\lambda^{2} \boldsymbol{L}^{T} \boldsymbol{L}\right)^{-1} \times\left(\boldsymbol{A}^{\boldsymbol{T}} \boldsymbol{C}\right)
$$

Therefore, the release rate $\boldsymbol{q}$ can be obtained once the response matrix $\boldsymbol{A}$, the regularization parameter $\lambda$ and the regularization matrix $\boldsymbol{L}$ were determined. Based on Equation (7), the discrete format of the concentration response at a certain point can be expressed as:

$$
\begin{aligned}
c_{t_{n}}= & \sum_{k=0}^{n} q_{t_{n-k}} F_{t_{k}}=q_{t_{n}} F_{t_{0}}+\cdots+q_{t_{n-k}} F_{t_{k}}+\cdots+ \\
& q_{t_{0}} F_{t_{k}}
\end{aligned}
$$

where $c_{t_{n}}$ is the concentration at $t_{n}$ time at a location, $q_{t n}$ is the release rate at $t_{n}$ time, and $F_{t_{n}}$ is the response factor. In the steady flow field, response factor is the concentration response for the unit impulse release, which can be obtained by calculating the monitored contaminant concentration at $t_{n}$ time; The response matrix $\boldsymbol{A}$ can be expressed as Equation (10) in terms of concentration response factors, and it can be obtained by MATLAB.

$$
\boldsymbol{A}=\left[\begin{array}{cccccc}
F_{t_{0}} & 0 & \cdots & \cdots & \cdots & 0 \\
F_{t_{1}} & F_{t_{0}} & 0 & \cdots & \cdots & 0 \\
\vdots & \ddots & \ddots & \ddots & \ddots & \vdots \\
F_{t_{k}} & F_{t_{k-1}} & \cdots & F_{t_{0}} & \cdots & 0 \\
\vdots & \ddots & \ddots & \ddots & \ddots & \vdots \\
F_{t_{n}} & F_{t_{n-1}} & \cdots & F_{t_{n-k}} & \cdots & F_{t_{0}}
\end{array}\right] \in \boldsymbol{R}^{(n+1) \times(n+1)}
$$

The most popular form of $\boldsymbol{L}$ is of the second derivative like format as Equation (11), because in that case gives the best combination of good balance to the data and the smooth solution (Kathirgamanathan et al., 2003):

$$
\boldsymbol{L}=\left[\begin{array}{cccccccc}
1 & -2 & 1 & 0 & \cdots & \cdots & \cdots & 0 \\
0 & 1 & -2 & 1 & \ddots & \ddots & \ddots & \vdots \\
\vdots & \ddots & \ddots & \ddots & \ddots & \ddots & \ddots & \vdots \\
\vdots & \ddots & \ddots & 1 & -2 & 1 & \ddots & \vdots \\
\vdots & \ddots & \ddots & \ddots & \ddots & \ddots & \ddots & 0 \\
0 & \cdots & \cdots & \cdots & 0 & 1 & -2 & 1
\end{array}\right] \in \boldsymbol{R}^{(n-2) \times n}(11)
$$

The regularization parameter $\lambda$ controls the weight given to minimization of the regularization term relative to minimization of the residual norm. A small $\lambda$ means that the solution error is mainly caused by the error of the input data, while a large $\lambda$ means that the error of the solution is mainly dominated by the error of the regularization term (Hansen, 1994). Therefore, choosing a suitable regularization parameter $\lambda$ is significant for achieving an accurate release rate $\boldsymbol{q}$. Three widely used methods for estimating regularization parameter are the L-Curve, Generalized cross-validation (GCV) criterion and Quasioptimality (Quasiopt) criterion. The specific principles of 
the three methods are detailed in Regularization Tools (Hansen, 1994) and these methods can be realized by MATLAB.

\section{Solution strategies}

The first step of this method is to partition the building and build pollutant transition probability matrix through Markov chain model, then the second step is to quantify the source strength of potential sources through Tikhonov regularization method with three different methods. In this study, we used the multi-zone model (CONTAM) validated by experimental data in a multi-compartment apartment to test the method. Figure 1 shows the specific layout of the apartment consisting of seven rooms, and $\mathrm{CO}_{2}$ as the tracer gas was released in Bedroom 2, the source release rate is $5 \mathrm{~L} / \mathrm{min}$ and the air supply rate is 680 $\mathrm{m}^{3} / \mathrm{h}$. The flow field and concentration distribution were predicted by CONTAM simulation and agreed well with the experimental data. More detailed information can be found in the study of (Zhai et al., 2012).

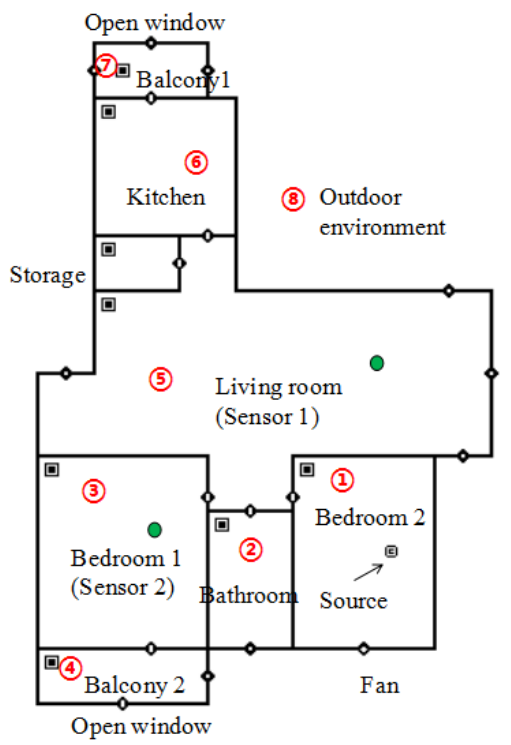

Figure 1 Multi-zone airflow model

\section{Results}

This model contains a steady-state continuous released source and fixed air supply rate. The carbon dioxide was released in Bedroom 2 with $5 \mathrm{~L} / \mathrm{min}$ release rate, the supply air rate is $680 \mathrm{~m}^{3} / \mathrm{h}$, and the concentration of the two sensors (Living room and Bedroom 1) within initial $1500 \mathrm{~s}$ was recorded to estimate the source release rate. The simulation concentration values were firstly validated by experimental data, and the detailed comparison was illustrated in Figure 2.

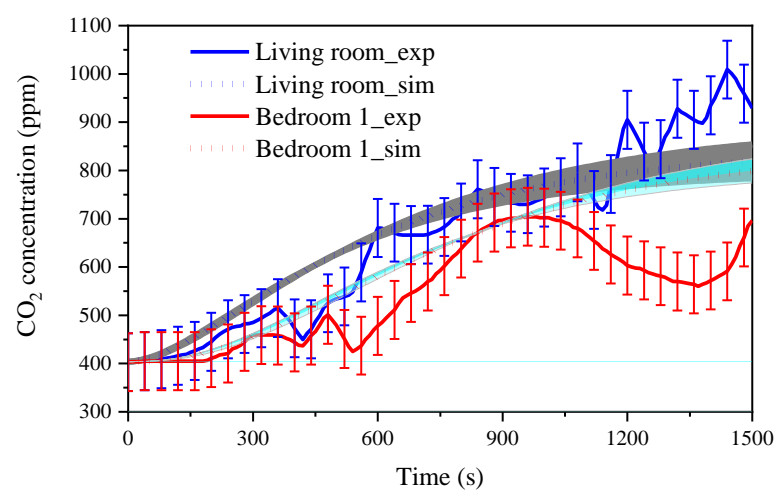

Figure 2: Comparison of the measured and multizone simulated CO2 concentrations in Living room and Bedroom 1 of the apartment

Figure 2 also shows the $10 \%$ concentration error of simulated data due to the actual supply air rate from the fan. Considering the accuracy of the $\mathrm{CO}_{2}$ sensors used is $\pm 40 \mathrm{ppm}+3 \%$ of reading, which leads to the reading uncertainty of about $60 \mathrm{ppm}$, then the $\mathrm{CO}_{2}$ concentrations simulated by CONTAM agree reasonably well with those obtained from the experiment. Therefore, the simulated values can be used to estimate the source release rate. The inverse process can be divided into two categories according to concentration data from Sensor 1 or from Sensor 2. At the same time, we compared the performances of three different methods solving the regularization parameter $\lambda$ in Tikhonov method. The inverse results were shown in Figure 3 and Figure 4.

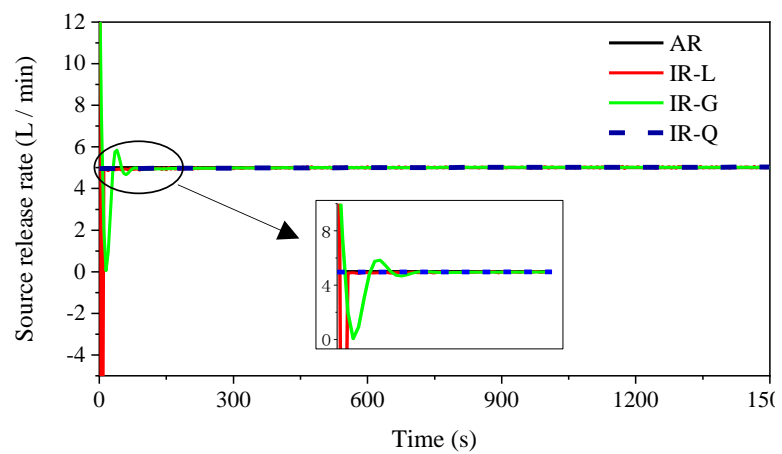

(a) $\Delta t=5 \mathrm{~s}$

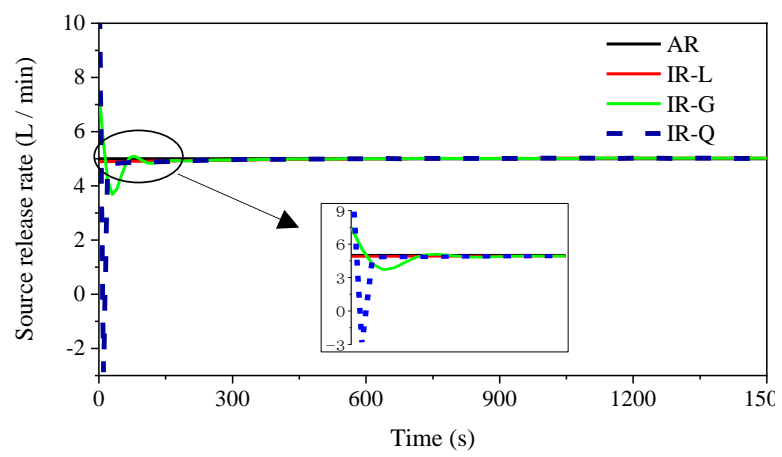

(b) $\Delta t=10 \mathrm{~s}$ 


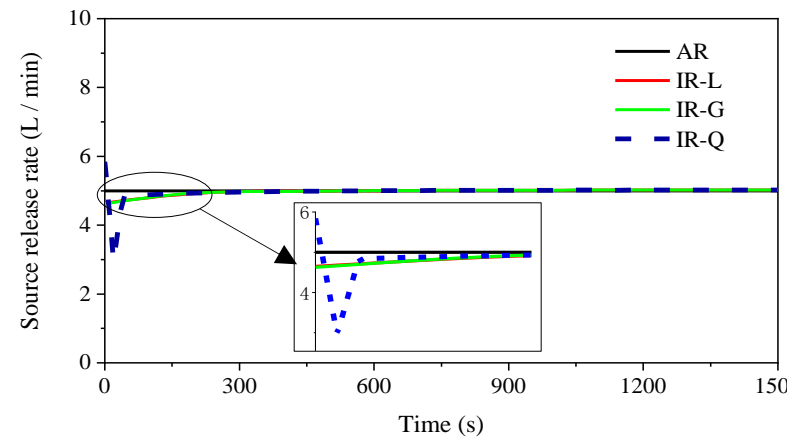

(c) $\Delta t=20 \mathrm{~s}$

Figure 3: Difference comparison of time resolution with Sensor 1 to estimate the source strength (AR is the actual release rate in Bedroom 2, IR represents the inverse release rate, and $L, G$ and $Q$ displays the inverse results by L-Curve method, GCV method, and Quasiopt method, respectively)

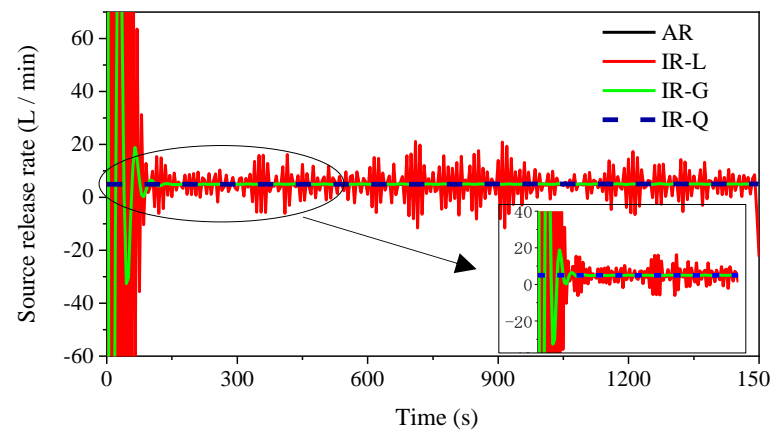

(a) $\Delta t=5 \mathrm{~s}$

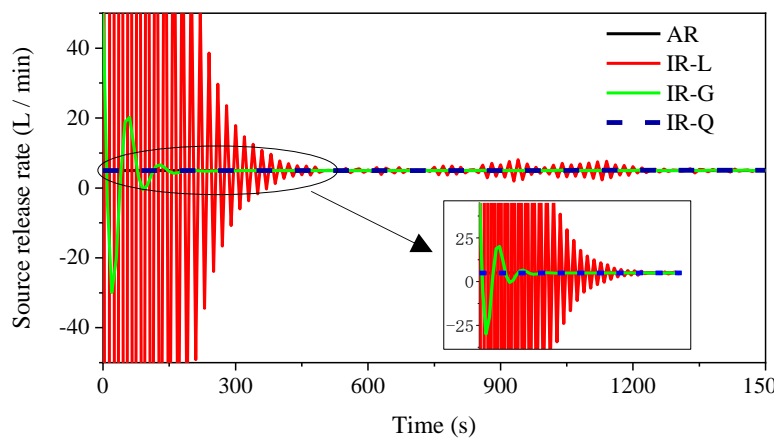

(b) $\Delta t=10 \mathrm{~s}$

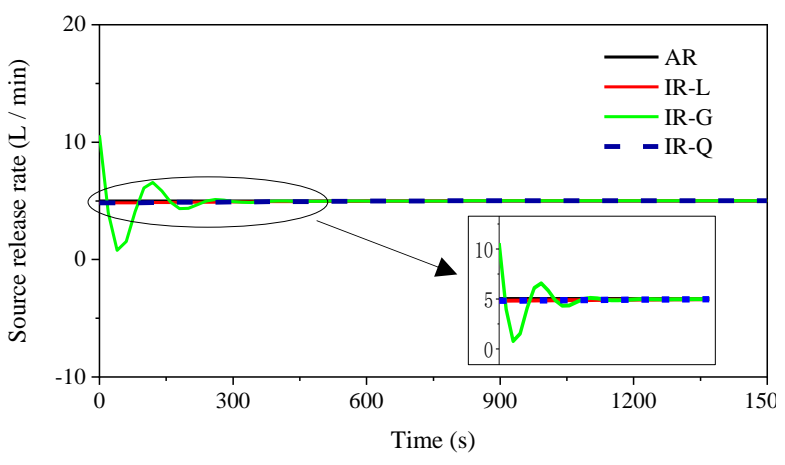

(c) $\Delta t=20 \mathrm{~s}$

Figure 4: Difference comparison of time resolution with Sensor 2 to estimate the source strength (AR is the actual release rate in Bedroom 2, IR represents the inverse release rate, and $L, G$ and $Q$ displays the inverse results by L-Curve method, GCV method, and Quasiopt method, respectively)

Figure 3 and Figure 4 exhibits the release rate quantification for the source in the Bedroom 2 by using Sensor 1 and Sensor 2 with different time step and different regularization methods, respectively. Relative error was introduced to quantify the inverse identification errors between the inverse rate profiles and actual ones (Wei et al., 2017):

$$
\text { error }=\frac{\left|N_{I R}-N_{A R}\right|}{N_{A R, p e a k}}
$$

where $N_{I R}$ is the inverse release rate, $N_{A R}$ is the actual release rate, and $N_{A R \text {, peak }}$ is the peak rate of the actual profile, $N_{A R \text {, peak }}=5$ is adopted. The ratio of time nodes at which relative error equals $10 \%$ to total time nodes was set as the threshold. Table 1 shows the proportion below this threshold for Sensor 1 and Sensor 2 under different time resolution (the data in Table 1 are the average values from three parameter choice methods under same time step).

Table 1: Performance of different sensors under three time resolutions.

\begin{tabular}{|c|c|c|}
\hline & Sensor 1 & Sensor 2 \\
\hline $5 \mathrm{~s}$ & $98.89 \%$ & $65.78 \%$ \\
\hline $10 \mathrm{~s}$ & $98.23 \%$ & $74.14 \%$ \\
\hline $20 \mathrm{~s}$ & $99.12 \%$ & $96.05 \%$ \\
\hline
\end{tabular}

It shows that the inverse result by Sensor 1 is better than that by Sensor 2 under each time step, especially under 5 $\mathrm{s}$. This is because Sensor 1 is more closed to the emission source in Bedroom 2.

The inverse results of three regularization parameter choice methods under different time resolutions are shown in Table 2 (the data in Table 2 are the average values from two sensors under same time step).

Table 2: Performance of different methods under three time resolutions.

\begin{tabular}{|c|c|c|c|}
\hline & L-Curve & GCV & Quasiopt \\
\hline $5 \mathrm{~s}$ & $52.33 \%$ & $94.68 \%$ & $100 \%$ \\
\hline $10 \mathrm{~s}$ & $64.90 \%$ & $94.37 \%$ & $99.34 \%$ \\
\hline $20 \mathrm{~s}$ & $100 \%$ & $94.08 \%$ & $98.68 \%$ \\
\hline
\end{tabular}

It is note that the percentage below the threshold of LCurve method is gradually increasing as the time step increases, while the other two methods show the opposite trend. The performance of L-Curve method is worse than the other two methods when time steps are $5 \mathrm{~s}$ and $10 \mathrm{~s}$. However, when time step is $20 \mathrm{~s}$, the performance of LCurve method is better than GCV method and Quasiopt method. This may be related to differences in their principles. The L-curve method is a logarithmic plot of residual norm versus the solution norm, and an optimal value can be found to be the point where this curve has maximum curvature. The principle of GCV mehod is similar to the least squares formulation, the value of the smoothing parameter that minimizes the predicted errors 
is the optimal one (Trujillo and Busby, 1997). This method is more suitable for the high frequency contents of the inverse problem. A geometric sequence of regularization parameters was considered in the Quasiopt method, and the corresponding regularized solution could be obtained, the optimal parameter is the one at which the difference norm of adjacent regularization solution is minimum. The advantage of this method is a comparably large independence of the underlying noise model. (Bauer and Kindermann, 2008).

To sum up, the determination of time solution should be according to actual inverse problem. The selection of regularization parameter for the high frequency contents of the inverse problem (large sample size) is more suitable by GCV method and Quasiopt method. On the contrary, the L-Curve method is more applicable for low frequency sampling.

\section{Conclusions}

In this study, we employed Tikhonov regularization to inverse the source emission rate in a multi-zone apartment based on the Markov Chain model. The model performances by choosing different regularization parameters (L-Curve, GCV, Quasiopt) and sensor locations under three time resoluions ( $5 \mathrm{~s}, 10 \mathrm{~s}$ and $20 \mathrm{~s}$ ) were compared. The following conclusions are drawn:

(1) Sensor 1 which was more closed to the source can provide more accuracy inverse emission rate than Sensor 2;

(2) For different time resolution, the performance of the parameter calculation method was different. The GCV method and Quasiopt method were more suitable for the inverse problem with a smaller sampling interval (high frequency sampling), while the L-Curve method was more applicable for the larger sampling interval.

\section{Acknowledgement}

This study was supported by the National Natural Science Foundation of China (Grant No. 51708286), the Natural Science Foundation of Jiangsu Province (Grant No. BK20171015), the Postgraduate Research \& Practice Innovation Program of Jiangsu Province (No. KYCX19_0854).

\section{References}

Alexander, D. and Klein, S. (2003). Biochemical terrorism: too awful to contemplate, too serious to ignore : subjective literature review. British Journal of Psychiatry 183(6), 491-7.

Bauer, F. and Kindermann, S. (2008). The quasioptimality criterion for classical inverse problems. Inverse Problems 24(3), 035002.

Chen, C. Liu, W. Lin, C. and Chen, Q. (2015). A Markov chain model for predicting transient particle transport in enclosed environments. Building and Environment 90, 30-36.

Chen, C. and Zhao, B. (2011). Review of relationship between indoor and outdoor particles: I/O ratio, infiltration factor and penetration factor. Atmospheric Environment 45(2), 275-288.

Chen, Y. Cai, H. Chen, Z. and Feng, Q. (2017). Using multi-robot active olfaction method to locate timevarying contaminant source in indoor environment. Building and Environment 118, 101-112.

Feng, Q. Cai, H. Li, F. Yang, Y. and Chen, Z. (2018). Locating time-varying contaminant sources in $3 \mathrm{D}$ indoor environments with three typical ventilation systems using a multi-robot active olfaction method. Building Simulation 11(3), 597-611.

Fontanini, A. Vaidya, U. and Ganapathysubramanian, B. (2015). Constructing Markov matrices for real-time transient contaminant transport analysis for indoor environments. Building and Environment 94, 68-81.

Hansen, P. C. (1994). REGULARIZATION TOOLS: A Matlab package for analysis and solution of discrete ill-posed problems. Numerical Algorithms 6(1), 1-35.

Hutchinson, M. Oh, H. and Chen, W. H. (2017). A review of source term estimation methods for atmospheric dispersion events using static or mobile sensors. Information Fusion 36, 130-148.

Kathirgamanathan, P. Mckibbin, R. and Mclachlan, R. I. (2003). Source release rate estimation of atmospheric pollution from a non-steady point source - Part 2: Source at an unknown location. Res. Lett. Inf. Math. Sci. 5, 85-118.

Kathirgamanathan, P. Mckibbin, R. and Mclachlan, R. I. (2004). Source Release-Rate Estimation of Atmospheric Pollution from a Non-Steady Point Source at a Known Location. Environmental Modeling \& Assessment 9(1), 33-42.

Li, Y. Huang, X. Yu, I. T. Wong, T. W. and Qian, H. (2010). Role of air distribution in SARS transmission during the largest nosocomial outbreak in Hong Kong. Indoor Air 15(2), 83-95.

Sohn, M. D. Reynolds, P. Singh, N. and Gadgil, A. J. (2002). Rapidly locating and characterizing pollutant releases in buildings. Journal of the Air \& Waste Management Association 52(12), 1422-1432.

Tikhonov, A. N. and Arsenin, V. Y. (1977). Solutions of ill-posed problems, Halsted Press. Washington (USA).

Trujillo, D M. and Busby, H. R. (1997). Practical Inverse Analysis in Engineering, CRC Press. Boca Raton (USA).

Vukovic, V. Tabares-Velasco, P. C. and Srebric, J. (2010). Real-Time Identification of Indoor Pollutant Source Positions Based on Neural Network Locator of Contaminant Sources and Optimized Sensor Networks. Journal of the Air \& Waste Management Association 60(9), 1034-1048.

Wei, Y. Zhou, H. Zhang, T. and Wang, S. (2017). Inverse identification of multiple temporal sources releasing the same tracer gaseous pollutant. Building and Environment 118, 184-195. 
Zhai, Z. Liu, X. Wang, H. Li, Y. and Liu, J. (2012). Experimental verification of tracking algorithm for dynamically-releasing single indoor contaminant. Building Simulation 5(1), 5-14.

Zhang, T. Zhou, H. and Wang, S. (2015). Inverse identification of the release location, temporal rates and sensor alarming time of an airborne pollutant source. Indoor Air 25(4), 415-427.
Zheng, X. and Chen, Z. (2011). Inverse calculation approaches for source determination in hazardous chemical releases. Journal of Loss Prevention in the Process Industries 24(4), 293-301.

Zou, Y. Luo, D and Chen, W. (2009) Swarm robotic odor source localization using ant colony algorithm. IEEE International Conference on Control and Automation. Christchurch (New Zealand), 9-11 December 2009. 